\title{
Potencialidades e desafios no exercício da preceptoria no estágio curricular supervisionado da graduação em enfermagem
}

\author{
Potentialities and challenges in the pratice of preceptorship in the supervised curricular \\ internship of nursing graduation
Potencialidades y desafíos en la práctica de la preceptoría en la pasantía curricular supervisada de graduación de enfermería

\begin{abstract}
Sandra Lúcia Dias Nunes ${ }^{1 *}$, Maria Alzira Rêgo Pinheiro ${ }^{1}$, Ana Elza Oliveira de Mendonça ${ }^{1}$, Marcelo Viana da Costa ${ }^{2}$, Ana Cristina Pinheiro Fernandes de Araújo.
\end{abstract}

\section{RESUMO}

Objetivo: Identificar potencialidades e desafios no exercício da preceptoria no estágio curricular supervisionado hospitalar da graduação em Enfermagem. Métodos: Estudo exploratório, descritivo de abordagem quali-quantitativa, realizado de maio a junho de 2018, em um Hospital Universitário do estado do Rio Grande do Norte. A coleta de dados envolveu respostas de um questionário de 21 enfermeiros preceptores. Para análise e interpretação dos dados, seguiram-se três etapas: pré análise das respostas, exploração do material e construção do discurso coletivo. Para análise estatística utilizou-se o software Alceste 4.9. Resultados: As experiências vivenciadas na preceptoria foram percebidas como satisfatórias pelos enfermeiros e os resultados foram agrupados em quatro categorias temáticas: potencialidades, dificuldades, sugestões e experiências. Os desafios identificados relacionaram-se, principalmente, a ausência da capacitação dos profissionais e protocolos para desenvolver a supervisão, a duplicidade de funções durante as atividades do preceptor e a desarticulação entre a instituição de ensino e serviço. Conclusão: A capacidade e a facilidade de comunicação entre preceptores e discentes destacaram-se como potencialidades para o exercício da preceptoria. Já à duplicidade de funções, à desarticulação entre instituição de ensino e serviço configuraram-se como desafios.

Palavras-chave: Preceptoria, Estudantes de enfermagem, Educação em saúde.

\begin{abstract}
Objective: To identify the potential and challenges in the exercise of preceptorship in the supervised hospital internship of undergraduate nursing. Methods: Exploratory, descriptive study with a qualitative and quantitative approach, carried out from May to June 2018, at a University Hospital in the state of Rio Grande do Norte. Data collection involved responses to a questionnaire from 21 nurse preceptors. For data analysis and interpretation, three steps were followed: pre-analysis of responses, exploration of material and construction of collective discourse. For statistical analysis, the Alceste 4.9 software was used. Results: The experiences in the preceptorship were perceived as satisfactory by the nurses and the results were grouped into four thematic categories: potentialities, difficulties, suggestions and experiences. The challenges identified were mainly related to the lack of training of professionals and protocols to develop supervision, the duplication of functions during the preceptor's activities and the disarticulation between the educational institution and service. Conclusion: The ability and ease of communication between preceptors and students was highlighted as potential for the exercise of preceptorship. Regarding the duplication of functions, the disarticulation between the educational institution and the service were challenges.
\end{abstract}

Keywords: Preceptorship, Nursing students, Health education.

${ }^{1}$ Universidade Federal do Rio Grande do Norte (UFRN), Natal - RN. *E-mail: sandrahuol@gmail.com

2Universidade Estadual do Rio Grande do Norte (UERN), Natal - RN.

SUBMETIDO EM: 9/2020

ACEITO EM: 10/2020

PUBLICADO EM: 1/2021 


\section{RESUMEN}

Objetivo: Identificar las potencialidades y desafíos en el ejercicio de la preceptoría en el internado hospitalario supervisado de pregrado en enfermería. Métodos: Estudio exploratorio, descriptivo con abordaje cualitativo y cuantitativo, realizado de mayo a junio de 2018, en un Hospital Universitario del estado de Rio Grande do Norte. La recolección de datos involucró respuestas a un cuestionario de 21 enfermeras preceptoras. Para el análisis e interpretación de los datos se siguieron tres pasos: preanálisis de respuestas, exploración de material y construcción de discurso colectivo. Para el análisis estadístico se utilizó el software Alceste 4.9. Resultados: Las experiencias en la preceptoría fueron percibidas como satisfactorias por los enfermeros y los resultados se agruparon en cuatro categorías temáticas: potencialidades, dificultades, sugerencias y experiencias. Los desafíos identificados se relacionaron con la falta de formación de profesionales y protocolos para desarrollar la supervisión, la duplicación de funciones durante las actividades del preceptor y la desarticulación entre la institución educativa y el servicio. Conclusión: La habilidad y facilidad de comunicación entre preceptores y alumnos se destacó como potencial para el ejercicio de la preceptoría. En cuanto a la duplicidad de funciones, la desarticulación entre la institución educativa y el servicio fueron desafíos.

Palabras clave: Preceptoría, Estudiantes de enfermería, Educación en salud.

\section{INTRODUÇÃO}

A palavra preceptor é atribuída àquele que orienta, oferece suporte, ensina, compartilha experiências e impulsiona a aquisição de conhecimentos científicos, dentro de uma concepção crítica e reflexiva do cotidiano do trabalho, bem como no desenvolvimento das habilidades do recém-graduado em sua atuação profissional (ROCHA PF, et al., 2016).

Na Enfermagem, a preceptoria iniciou em 1994 com o Parecer nํ14/94 do Conselho Federal de Educação e a Portaria do Ministério da Educação (MEC) o 1.721 de 15 de dezembro de 1994, que estabelecem o currículo mínimo, a supervisão e o acompanhamento dos alunos de graduação por profissionais de serviço (MELO POC, 2018; OGUISSO T, et al., 2019).

Nesse sentido, o estágio curricular supervisionado surgiu como uma forma de efetivar a articulação entre a formação acadêmica e a realidade profissional. Assim, o estudante tem a oportunidade de desenvolver as quatro competências do enfermeiro: cuidar, gerenciar, educar e pesquisar. Dentro da vivência do trabalho, ainda são estimulados outros valores, como a responsabilidade, autonomia, liberdade, criatividade, compromisso, domínio da prática e o papel social (TREVISO P, et al., 2017).

A importância do preceptor envolve a integração entre o ensino e o serviço e a teoria e a prática, contribuindo para a formação de profissionais competentes com capacidade de resolver demandas de saúde da população. No entanto, existem desafios a serem superados, a exemplo: sobrecarga de funções, déficit de capacitação, deficiência de articulação e ausência de padronização do acompanhamento dos discentes (RIBEIRO PKC, et al., 2020).

Observa-se que para o bom desempenho do preceptor no acompanhamento, é necessário clareza de seu papel, ampliando os conhecimentos e possibilitando entendimento ao aluno. Diante desse contexto, surgiu a inquietação de analisar a situação funcional desses enfermeiros, concebendo-se a seguinte questão de pesquisa: Quais as potencialidades e desafios no desempenho da preceptoria na formação em enfermagem? Para responder a este questionamento, traçou-se o objetivo: Identificar as potencialidades e desafios no exercício da preceptoria no estágio curricular supervisionado hospitalar da graduação em Enfermagem.

\section{MÉTODOS}

Trata-se de um estudo exploratório, descritivo de abordagem quali-quantitativa. A pesquisa foi realizada em um Hospital Universitário do estado do Rio Grande do Norte, primeira instituição de nível terciário da rede pública a servir de campo de práticas para a realização do estágio supervisionado do curso em Enfermagem. 
A população de estudo foi composta por enfermeiros que desempenham a função de preceptor do último período do curso, em enfermarias, há pelo menos um ano. Foram excluídos os profissionais que se encontravam ausentes dos serviços por afastamento e os que não cumprem escalas de plantões noturnos. Assim, a amostra foi composta por 21 enfermeiros.

A coleta de dados ocorreu nos meses de maio a junho de 2018, por meio de um questionário, composto por 13 perguntas, dividido em duas partes. A primeira refere-se à caracterização profissional e a segunda, composta por questões que buscaram responder, especificamente, ao objetivo do estudo.

As respostas foram analisadas pelo método de análise de conteúdo proposto por Bardin. Assim, na fase de análise e interpretação dos dados, seguiram-se três etapas: a pré-análise com leitura flutuante dos questionários, a exploração do material com a marcação dos trechos, frases e fragmentos de texto e, por fim, a construção de um discurso coletivo. Após a avaliação de três juízes, emergiram quatro categorias temáticas: potencialidades da preceptoria, dificuldades vivenciadas pelo preceptor, sugestões para melhorar o exercício da preceptoria e experiências pessoais na vivência da preceptoria.

Para a análise estatística utilizou-se o software Alceste 4.9, que consiste em uma metodologia de análise de dados qualitativos, que pretende tratar o material textual, sobretudo no que se refere à sua composição lexical e estruturação temática. O estudo atendeu aos preceitos da Resolução no 466/2012 do Conselho Nacional de Saúde (CNS). A coleta de dados teve início após a aprovação pelo Comitê de Ética em Pesquisa (CEP) do Hospital Universitário Onofre Lopes, sob o no 2.603.436, de 17/04/2018.

\section{RESULTADOS E DISCUSSÂO}

Quanto à caracterização dos enfermeiros preceptores, a maioria pertencia ao sexo feminino $(81,0 \%)$, com idade média de 35,0 e desvio padrão de 7,56. O tempo de formação variou de um a 35 anos, com média de 10,3 anos e desvio padrão de 7,01. Observou-se que 85,4\% apresentavam um e três anos de formados. Todos possuem pós-graduação a nível de especialização (61,9\%) e mestrado (38,1\%); Tal informação demonstra preocupação por parte desses profissionais em aprimoração e atualização dos conhecimentos.

Com relação ao tempo de atuação no serviço prevalece o tempo inferior a 10 anos e ao tempo de experiência como preceptor variou de um a 15 anos, com maior frequência de um a três anos, que correspondeu a $85,4 \%$, com média de 3,5 anos e desvio padrão de 3,9. Notabilizou-se que $61,9 \%$ dos enfermeiros nunca participaram de cursos voltados para atuação em preceptoria.

Após a análise dos discursos, emergiram quatro categorias temáticas intituladas: potencialidades da preceptoria, dificuldades vivenciadas pelo preceptor, sugestões para melhorar o exercício da preceptoria e experiências pessoais na vivência da preceptoria, descritas a seguir.

\section{Categoria 1 - Potencialidades da preceptoria}

As potencialidades identificadas direcionaram paras as seguintes competências: Trabalho em equipe; comunicação; gerência; resolução de problemas; promoção da saúde; atenção à saúde e valores profissionais. Destaca-se também a capacidade e facilidade de comunicação entre preceptores e discentes. Essas qualidades são fundamentais para o compartilhamento dos conhecimentos, como evidenciadas nas falas:

"Boa comunicação e facilidade de articulação com outros profissionais; Habilidade técnica; Conhecimento científico". (preceptor 1)

"Objetividade na hora de realizar procedimentos, boa comunicação. Agilidade e dinâmica durante o trabalho. Estímulo dos estudantes a estudar e realizar raciocínio crítico/científico". (Preceptor 2)

A comunicação é o elemento central que interliga todo processo de trabalho e parte de uma construção coletiva do conhecimento, resultado do encontro de diferentes atores sociais e seus determinantes de saúde e de adoecimento. Em consonância, o preceptor apresentou papel relevante de orientar, facilitar e mediar o processo de ensino-aprendizagem, utilizando-se da comunicação efetiva (ROCHA PF, et al., 2016). 
O preceptor é percebido como o ator/atriz que contribuirá para o aprimoramento dessas competências. No entanto, é necessário que o discente possua determinadas competências para exercer a função, conforme nos trechos dos preceptores abaixo:

\author{
"Habilidade técnica; Conhecimento científico". (Preceptor 1) \\ "Iniciativa, Capacidade teórico-prática no campo da enfermagem". (Preceptor 3)
}

\begin{abstract}
Além das competências que os discentes devem apresentar, os profissionais tornam-se estimuladores e facilitadores no processo de aprendizado e desenvolvimento do discente em suas atividades durante todo 0 estágio. O preceptor é visto como um exemplo de carreira profissional do discente e deve apresentar boa condutas técnicas e éticas (BARROS MAA, et al., 2017).
\end{abstract}

Segundo o estudo de Muller G, et al. (2018), a preceptoria, em conjunto com os docentes, resultou em efeitos positivos na formação dos discentes. Observou-se melhora no desempenho quanto à autoconsciência, à autoeficácia e ao sucesso da aprendizagem. As discussões entre docente, discente e preceptor obtiveram impacto positivo no trabalho e na atuação do aluno. E para melhorar esse desempenho, existem modelos de supervisão de estágios capazes de organizar e melhorar as potencialidades dos discentes neste momento e que podem ser discutidas e analisadas em estágios nos diversos modelos de atenção à saúde (ESTEVES LSF, et al., 2019).

\title{
Categoria 2 - Dificuldades para exercer a preceptoria
}

O exercício da preceptoria é essencial para o desenvolvimento das habilidades e competências necessárias ao exercício da profissão. Contudo, é uma atividade que apresenta desafios que necessitam ser superados (RIBEIRO PKC, et al., 2020). A escolha da profissão é um momento delicado na vida de um indivíduo. Por fatores diversos, como imaturidade ou pressão familiar, a decisão torna-se mais complicada. $\mathrm{Na}$ finalização do curso, o aluno pode se deparar com conflitos pessoais em relação à sua profissão, prejudicando a postura do mesmo e o desempenho acadêmico, conforme a fala abaixo (FRACALOZZI NMN, et al., 2019; LIMA RS, et al., 2020):

"Alunos desinteressados, carência de um roteiro bem estabelecido para o período de estágio; por exemplo: programação de intervenção educativa e apresentação de relatório final". (Preceptor 4)

No estudo de Tavares P, et al. (2016), desenvolvido em um Hospital Universitário de Alagoas, os profissionais não se sentiam confortáveis em assumir a preceptoria, uma vez que consideravam a atividade mais uma demanda. Comparando com este estudo, observou-se que, em sua maioria, existe grande empenho por parte dos profissionais entrevistados em colaborar com o estágio curricular supervisionado. No entanto, percebeu-se que também existia o sentimento de ausência de valorização, somada à dificuldade no relacionamento com os alunos, devido à falta de compromisso dos alguns estudantes, observado nos discursos:

"Acredito que o fator tempo seja o mais limitante para que se possa exercer o papel de preceptor, uma vez que as responsabilidades assistenciais, burocráticas, entre outras do enfermeiro, não são amenizadas quando da presença de alunos sob sua supervisão. Também sinto falta de momentos de aperfeiçoamentos exercer tal papel com maior excelência". (Preceptor 5)

"Ao exercermos a preceptoria e assumimos as enfermarias, isso dificulta, uma vez que já temos a responsabilidade com o paciente já o setor e aumentamos a sobrecarga com o ensino". (Preceptor 6)

Nos estudos de Andrade SR, et al. (2015), Ponte Neto AO, et al. (2017) e Miolo SB, et al. (2020), esta realidade da carga horária extra e do acúmulo de atividades para o exercício da preceptoria também está presente. Compreende-se que a construção do conhecimento é um processo que não se realiza de forma rápida. 
É necessário tempo para que o preceptor acompanhe, compartilhe e estimule criticidade na prática dos discentes. Tais estudos estimulam à existência de carga horária específica para os preceptores desenvolverem e participarem das preceptorias. Esta ação pode incentivar o profissional a ter um tempo previsto para planejar e executar ações com o discente, melhorando a atuação e aprendizado (MIOLO SB, et al., 2020).

\section{Categoria 3 - Sugestões}

O enfermeiro ocupa uma posição estratégica nos serviços de saúde, assumindo funções assistenciais, gerenciais e educativas. Dentre as educativas, destacam-se as atividades de preceptoria. Sendo assim, as atividades de ensino e assistência ocorrem simultaneamente (NEVES MGBC, et al., 2020). Este volume de atribuições do enfermeiro impactou negativamente no acompanhamento oferecido aos discentes, como relatado abaixo por um preceptor:

"O nosso maior desafio é não conseguir dar total atenção que gostaria ao estudante, em virtude de nossas atividades assistenciais, burocráticas e administrativas". (Preceptor 2)

É importante que o preceptor domine saberes do seu campo de prática, como também compreenda pedagogicamente como transmitir este conhecimento ao aluno. No entanto este aprendizado não deve transcorrer mediante a repetição mecânica de fazeres, mas deve envolver uma perspectiva crítica e reflexiva do cotidiano do trabalho, capaz de promover transformações nas pessoas, nas práticas e nas instituições. Nesse sentido, faz-se necessária a capacitação dos profissionais para esta atuação em preceptoria (NEVES MGBC, et al., 2020).

A Universidade tem papel fundamental na formação dos preceptores, favorecendo a participação em cursos de atualização nas áreas específicas e em eventos científicos. Entretanto, os preceptores revelaram aspectos negativos em relação à distância entre o serviço e a academia e a inexistência de um instrumento padronizado para acompanhamento dos discentes (SILVA HGN, et al., 2020).

"Acho injusto e até mesmo errado o distanciamento entre os professores do campus e os preceptores, acho que deixam os alunos muito soltos, somente sob nossa responsabilidade, quando temos inúmeras outras atividades para desenvolver, muitas vezes não há tempo hábil para observar o aluno mais de perto". (Preceptor 7)

"Distância da academia e conciliar ensino serviço". (Preceptor 8)

"A ausência de instrumentos para subsidiar a prática; a não padronização dessa atitude entre os setores e ainda a falta de educação continuada para os preceptores. Ainda a falta de integração entre teoria e prática". (Preceptor 9)

"A criação de um pop ou instrumento de apoio para nortear os preceptores visando a padronização do trabalho bem como a integração dos conteúdos inerentes da enfermagem com a prática cotidiana". (Preceptor 9)

A partir da necessidade identificada pelos enfermeiros, foi proposto um roteiro para acompanhamento dos discentes da graduação em Enfermagem em Estágio Curricular, no qual foram descritas sete etapas a serem seguidas no decorrer do estágio, são elas: embasamento teórico e prático do aluno, unificar condutas, inserir o aluno na rotina do setor de trabalho, estimular a autonomia do aluno, utilizar a avaliação por meio do feedback diário, promover raciocínio clínico através de discussões e participar de encontros entre docentes, preceptores e alunos.

A formação dos profissionais de saúde deve responder às demandas do SUS e envolver uma atuação que tenha como princípios, as necessidades sociais e de saúde dos usuários e famílias (RIBEIRO PKC, et al., 2020). Contudo, a desarticulação entre as instituições formadoras e os serviços de saúde acentua o distanciamento entre a formação voltada para necessidades do SUS.

"Acho injusto e até mesmo errado o distanciamento entre os professores do campus e os preceptores, acho que deixam os alunos muito soltos, somente sob nossa 
responsabilidade, quando temos inúmeras outras atividades para desenvolver, muitas vezes não há tempo hábil para observar o aluno mais de perto". (Preceptor 7)

"Maior diálogo entre os cursos de formação com o serviço; pontuar os objetivos de aprendizagem em serviço para podermos direcionar melhor as atividades práticas dos alunos; minicursos de formação de preceptores para nos auxiliar a direcionar melhor os alunos durante sua formação". (Preceptor 10)

O modelo de ensino no Brasil é baseado na crença de que quem sabe, automaticamente, sabe ensinar. Todavia, a função de preceptoria ultrapassa esse fundamento. Sendo assim, é importante que o preceptor domine os saberes de seu campo de prática, como também domine na pedagogicamente o ensino e a aprendizagem no serviço, isto é, traga criticidade ao trabalho, capaz de promover transformações em sua realidade (FERREIRA FC, et al., 2018; PEIXOTO LS, et al., 2014).

Sendo assim, outro fator importante encontrado foi a ausência de formação para o processo de supervisão dentro das Universidades ou instituições, reconhecido pelo discurso dos sujeitos das pesquisas abaixo. Observou-se uma preocupação dos enfermeiros com a formação acadêmica dos alunos, contudo, ao iniciar as primeiras preceptorias, surgiram preocupações e anseios. Desse modo, para quebrar esta barreira, incentiva-se que as Universidades incluam em sua grade curricular uma disciplina direcionada à capacitação dos futuros formadores.

O investimento na formação dos preceptores também faz parte das políticas indutoras dos Ministérios da Saúde e da Educação há pelo menos uma década. O interesse em conhecer com profundidade o papel do preceptor já foi alvo de estudos e apontou a necessidade de investimentos por meio da educação permanente focada na interdisciplinaridade (ALBIEIRO JFG, et al., 2017).

"Necessidade de formação específica para preceptoria". (Preceptor 11)

"Atribuições burocráticas fora do escopo da enfermagem em excesso no setor, dificultando a discussão de casos e acompanhamento dos residentes. Falta de capacitação direcionadas ao exercício da preceptoria". (Preceptor 1)

Magalhães MSC, et al., (2020) identificaram a necessidade de formação para atuar na preceptoria ao realizar pesquisa com enfermeiros da atenção primária a saúde. Esse achado, enaltece a importância do conhecimento de metodologias de ensino e o desenvolvimento de habilidades necessárias para atuar positivamente na formação do futuro profissional.

\section{Categoria 4 - Experiência da preceptoria}

Nesta categoria, destaca-se a satisfação dos enfermeiros com as oportunidades de ensino, principalmente, em decorrência ao contato com os discentes que os potencializa a uma intensa troca de saberes e estímulos à busca do conhecimento e a atualização profissional.

O objetivo final do estágio curricular supervisionado é formar profissionais qualificados. Isso implica em uma assistência de qualidade e eficiente. Na experiência de preceptoria, o enfermeiro sente a necessidade de manter-se atualizado com as práticas profissionais e a participação do discente, em fase de conclusão de curso, é indispensável para esta troca de vivências e saberes.

"A experiência como preceptor é ao mesmo tempo enriquecedora e desafiadora, porque é necessário estarmos em constante atualização para atendermos as necessidades de aprendizagem e as indagações dos alunos, além disso também somos responsáveis por promover discussões e reflexões sobre nossas condutas, relacionadas ao paciente, a equipe e aos alunos". (Preceptor 10)

"Ser preceptor é uma experiência que exige bastante responsabilidade e segurança. Configura-se um período de amadurecimento, troca de saberes e adaptação mútua, que contribui para o crescimento pessoal e profissional tanto do aluno quanto do preceptor". (Preceptor 5) 


\begin{abstract}
"É uma experiência enriquecedora, porque ótima oportunidade de compartilhamento de conhecimentos, no qual a gente ensina, mas também aprende". (Preceptor 12)

"Boa, pois ao acompanhar os alunos sinto que eles têm muito a ensinar. Aprendo com eles. Temos que nos mantermos atualizados, para sermos preceptores. O que nos força a estudar para ensinarmos". (Preceptor 13)
\end{abstract}

Assim, o preceptor desenvolve um papel essencial na formação do aluno, sendo um elo de ligação entre teoria e prática. Recai sobre o mesmo a responsabilidade de receber o aluno e inseri-lo no ambiente de trabalho. Neste contexto, segundo Patrícia PAB, et al. (2015), à medida que os enfermeiros se sentem satisfeitos nas atividades de preceptoria, tornam-se mais estimulados a atualizações e a participação na educação permanente, possibilitando novos conhecimentos e troca de experiências com o graduando, confirmada pelas falas:

"É uma experiência enriquecedora, porque ótima oportunidade de compartilhamento de conhecimentos, no qual a gente ensina, mas também aprende". (Preceptor 12)

"Boa, pois ao acompanhar os alunos sinto que eles têm muito a ensinar. Aprendo com eles. Temos que nos mantermos atualizados, para sermos preceptores. O que nos força a estudar para ensinarmos". (Preceptor 13)

Para Fontes FLL, et al. (2019) os programas de pós-graduação deveriam incluir disciplinas nos currículos voltadas a formação do enfermeiro preceptor, uma vez que, o ensino não acontece somente em espaços acadêmicos, mas também em serviços de atenção a saúde. Os autores ressaltaram que a carga horária das disciplinas dos cursos para enfermeiros é centrada no cuidado clínico, restando pouco ou nenhum espaço de tempo para estimular a reflexão sobre as atividades de ensino. Zamprogna KM, et al. (2019) destacam que em muitos programas de pós-graduação as disciplinas voltadas ao ensino são optativas, possibilitando ao enfermeiro concluir sem ter nenhuma carga horária específica para o exercício prático da docência.

Por fim, percebe-se que as diversas experiências vivenciadas na preceptoria foram percebidas como satisfatórias pelos enfermeiros. Porém, foram constatadas adversidades durante a execução desta atividade, sendo as principais a ausência de capacitação dos profissionais, ausência de protocolos para desenvolver a supervisão e conflito de atividades pelo enfermeiro preceptor. É importante o debate e a reflexão acerca da preceptoria, uma vez que é nesta ação que ocorre a passagem do estudante para o profissional.

\title{
CONCLUSÃO
}

A atuação dos enfermeiros no exercício da preceptoria de discentes de graduação é permeada por desafios como à duplicidade de funções, à desarticulação entre a instituição de ensino e o serviço de saúde, à necessidade de formação específica para atuar na preceptoria e à ausência de padronização para acompanhamento e avaliação dos discentes. Como potencialidade destacou-se a capacidade e a facilidade de comunicação entre preceptores e discentes. Espera-se que os resultados desse estudo possam subsidiar o pensamento crítico e a reflexão dos profissionais sobre o seu papel no exercício da preceptoria, e ainda fomentar o desejo de mudanças que qualifiquem cada vez mais a atuação do enfermeiro.

\section{REFERÊNCIAS}

1. ALBIEIRO JFG, FREITAS SFT. Practice settings in primary health care: typology for teaching-care units. Rev. Bras Promoção Saúde, 2017; 30(3).

2. ANDRADE SR, et al. Perceptions of academic and practitioner nurses regarding the teaching-care partneship in primary healthcare units. Rev. interface, 2015, 19(54).

3. BARROS MAA, et al. Perfil acadêmico do preceptor de enfermagem na atenção primária à saúde. Rev. Expressão Cat Saúde, 2017; 2(2).

4. ESTEVES LSF, et al. Clinical supervision and preceptorship/tutorship: contributions to the supervised curricular internship in nursing education. Rev. Bras Enferm, 2019; 72(6). 
5. FERREIRA FC, et al. Saberes e competências do enfermeiro para preceptoria em unidade básica de saúde. Rev. Bras Enferm, 2018; 71(4).

6. FRACALOZZI NMN, et al. Professional maturity as a diagnosis of career education needs. Rev. Psicol Argum, 2019; 37(97).

7. LIMA PAB, ROZENDO CA. Challenges and opportunities in the Pró-PET-Health preceptorship. Rev. Interface, 2015; 19.

8. LIMA RS et al. Construction of professional identity in nursing students: qualitative research from the historical-cultural perspective. Rev. Latino-Am. Enferm, 2020; 28.

9. MELO POC. A formação do enfermeiro para atuar com a pessoa idosa na estratégia saúde da família. Dissertação (Mestrado em Enfermagem). Universidade Federal de Alagoas, 2018, 86.

10. MIOLO SB, FEDOSSE E. Tutoring and preceptory in multiprofessional residence programs in basic care: a necessary dialogue. Rev. Research, Society and Development, 2020; 9(3).

11. MUELLER G, et al. Quality assurance of the clinical learning environment in Austria: Construct validity of the clinical learning environment, supervision and nurse teacher scale (CLES + T scale). Rev. Nurse Educ Today, $2018 ; 66$.

12. NEVES MGBC, et al. The conceptions of tutors of the SUS on active methodologies in training of the health professional. Rev. Educ., 2020; 36.

13. OGUISSO T, et al. Historia de la enseñanza de historia de la enferméria em Brasil. Rev científica de la associación de Historia y Antropología de los Cuidados, 2019; 55.

14. PEIXOTO LS, et al. Research about the knowledge and teaching practice of the preceptor: a test pilot. Rev. enferm UFPE online, $2014 ; 8(7)$.

15. PONTE NETO OA, et al. Self-evaluation as an educational strategy in the context of multiprofessional residence program in family health and mental health. Rev. Tempus, actas de saúde colet, 2016, 10(4).

16. RIBEIRO PKC, et al. Health professional and a practice of preceptorship of primary care: assistance, training and possible transformations. Journal of Management \& Primary Health Care, 2020; 12.

17. ROCHA PF, et al. Preceptoria como modalidade de ensino na saúde: atuação e características do preceptor cirurgiãodentista da atenção primária. Rev Saberes plurais, 2016; 1.

18. SILVA HGN. The social role of the University through teaching-service-community integration in Brazil: systematic review and meta-synthesis. Rev. Linhas Críticas, 2020; 26.

19. TAVARES PEN, et al. A vivência do ser enfermeiro e preceptor em um hospital escola: olhar fenomenológico. Rev. Rene, 2011; 12(4).

20. TREVISO P, et al. Nursing skills in care management. Rev Adm Saúde, 2017; 17(69).

21. FONTES FLL, et al. Práticas pedagógicas usuais do enfermeiro docente para superação do modelo tradicional de ensino. Revista Eletrônica Acervo Saúde, 2019; (23): e249.

22. ZAMPROGNA KM, et al. Characterization of didactic and pedagogical training in Brazilian stricto sensu Postgraduate Programs in Nursing. Rev Esc Enferm USP, 2019; 53: e03430.

23. MAGALHÃES MSC, et al. Contributions of Nursing Preceptorship in the context of Primary Health Care from the perspective of active methodologies. Research, Society and Development, 2020; 9(7), e270973681. 